\title{
Controlled transition between parametric and Raman oscillations in ultrahigh- $Q$ silica toroidal microcavities
}

\author{
Bumki Min, ${ }^{\text {a) Lan Yang, and Kerry Vahala) }}$ \\ Thomas J. Watson Laboratory of Applied Physics, California Institute of Technology, \\ Pasadena, California 91125
}

(Received 28 April 2005; accepted 6 September 2005; published online 28 October 2005)

\begin{abstract}
A controllable and reversible transition between parametric and Raman oscillations in an ultrahigh$Q$ silica toroidal microcavity is experimentally demonstrated and theoretically analyzed. By direct change of cavity loading and indirect adjustment of frequency detuning, parametric and/or Raman oscillation can be accessed selectively without modification of cavity geometry in a toroidal microcavity with a large enough aspect ratio. Based on an effective cavity gain theory, this transition is analyzed in terms of cavity loading and frequency detuning leading to a better understanding of the combined effects of parametric and Raman processes in silica microcavities. (C) 2005 American Institute of Physics. [DOI: 10.1063/1.2120921]
\end{abstract}

Toroidal microcavity resonators ${ }^{1}$ have extended the performance of well-known microsphere resonators (including liquid microdroplets) by providing both a simplified resonator spectrum and ultrahigh- $Q$ performance on a silicon microelectronic chip. Like microspheres they provide a combination of an effectively long-interaction length (high-quality factor) and a microscale mode volume so that even weak nonlinear mechanisms, such as stimulated Raman scattering, are observable at only tens of microwatts of input power, which is orders of magnitude lower than corresponding threshold powers for fiber-based Raman lasers. ${ }^{2}$ However, in contrast to microsphere resonators, microtoroids have been proven to be more versatile by recently enabling the first demonstration of a Kerr-nonlinearity optical parametric micro-oscillator $(\text { micro-OPO })^{3}$ [note: a polariton-based micro-OPO in strongly coupled vertical cavity surfaceemitting laser (VCSEL) microcavities has also been reported previously]. ${ }^{4}$ Micro-OPOs are more challenging to realize than their stimulated counterparts as they require both ultrahigh- $Q$ performance and phase matching of the underlying nonlinear process. Of the two requirements for matching (momentum and energy conservations), momentum conservation is intrinsically satisfied in microcavities for symmetrically located signal and idler modes with respect to the pump mode. This is a natural consequence of the rotational symmetry which yields a propagation constant $\beta_{m}=2 m / D_{\text {eff }}$, where $m$ is an azimuthal mode number and $D_{\text {eff }}$ is an effective diameter of the light path in the cavity. Strict energy conservation, which is not satisfied automatically due to material and cavity mode dispersions, is relaxed by cross-phase modulation (and in part by self-phase modulation). The parametric gain bandwidth $\Omega$ gives the amount by which energy conservation can be relaxed in the process. ${ }^{3,5}$ This bandwidth which scales linearly with the pump power and the nonlinear coefficient is also progressively broadened by compressing the minor diameter $d$ (i.e., increasing the aspect ratio $D_{p} / d$ where $D_{p}$ is the principal diameter) of the toroidal microcavity, and thereby, in turn, the effective cross-sectional area of the mode. Empirically, $D_{p} / d>15$ has been proven sufficient

\footnotetext{
${ }^{\text {a) }}$ Electronic mail: bkmin@caltech.edu

b)URL: http://vahala.caltech.edu; electronic mail: vahala@ caltech.edu
}

to enable parametric oscillation. However, aspect ratio control is only a necessary condition and not a sufficient condition for parametric oscillations. In addition to this geometrical control, cavity loading provided by the waveguide used to couple power both to and from the resonator (and characterized by a coupling parameter $K=Q_{0} / Q_{\text {ex }}$, where $Q_{0}$ is the intrinsic quality factor and $Q_{\mathrm{ex}}$ is the external quality factor) should also be carefully adjusted. This dependence results because, as noted above, the parametric gain bandwidth scales with the circulating pump power in the cavity and this power can be controlled, in turn, by changing the coupling parameter $K$. Cavity loading variation should be able to induce transition between parametric and Raman oscillations in a sufficiently phase-matched silica toroidal microcavity. However, this loading-induced control of OPO and Raman oscillations has not been verified in previous experiments. ${ }^{3}$ In this paper, we demonstrate control of the oscillation re-
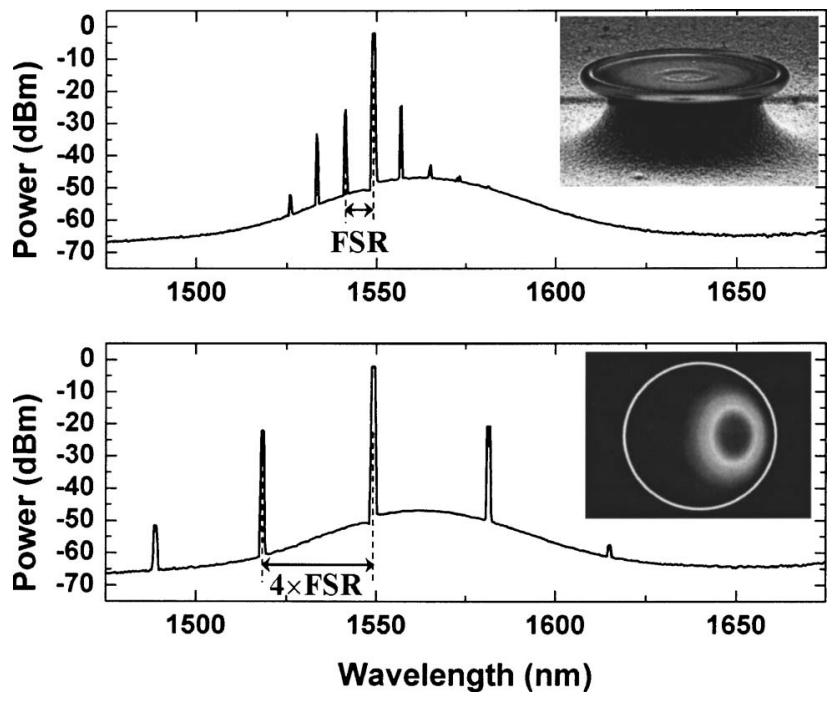

FIG. 1. (Color online) Two parametric oscillation spectra measured from the same ultrahigh- $Q$ silica toroidal microcavity with a minor $(d) /$ principal $(D)$ diameter of $3.8 / 67 \mu \mathrm{m}(D / d=17.63>15)$. The two spectra correspond to a different (horizontal/vertical) tapered fiber position with respect to the toroidal microcavity. A pump wavelength of $1549.2 \mathrm{~nm}$ is used for excitation. Insets: SEM micrograph (upper) and theoretical intensity pattern (lower) of the toroidal microcavity used for these spectra. 

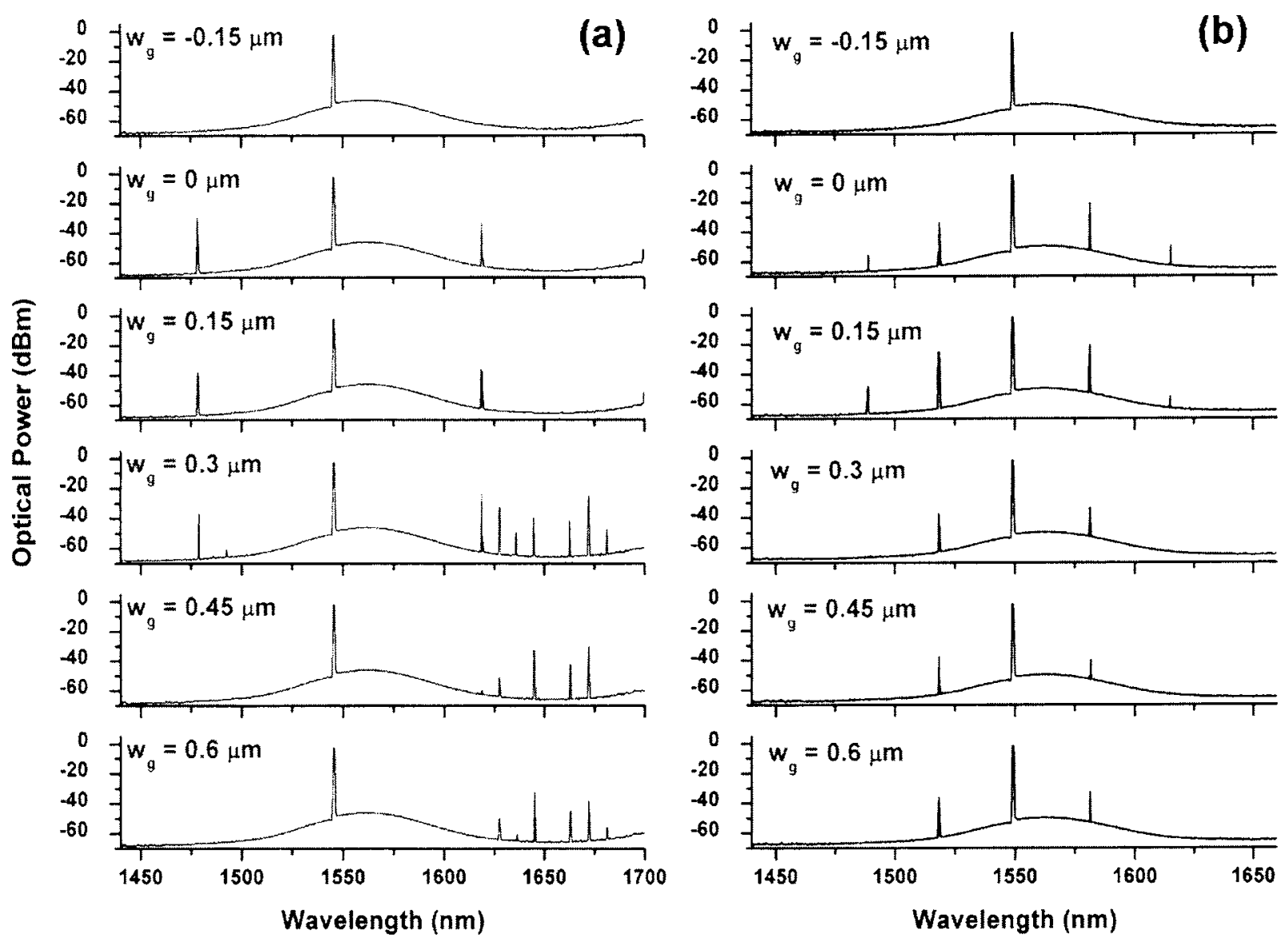

FIG. 2. (Color online) (a) Transition between Raman oscillation and parametric oscillation in a toroidal microcavity with larger frequency detuning $\Delta f$ $\sim 13 \mathrm{MHz}$. Cavity loading is different in each panel and is varied by adjustment of the air gap $\left(w_{g}\right)$. Air gap values are referenced to the critical coupling point (zero). (b) Toroidal microcavity with a smaller frequency detuning $\Delta f \sim 6 \mathrm{MHz}$ showing parametric-only regimes over a range of loading conditions. As in (a) cavity loading is controlled by adjustment of the taper-cavity gap distance shown in each plot and gap values are given relative to the critical-coupling gap distance. Loading increases in the vertical direction.

gime (Raman and parametric oscillations) by varying the cavity loading in a toroidal microcavity of a fixed, but sufficiently high, aspect ratio. The theoretical explanation for the transition of oscillations will be given in conjunction with actual experiments based on an effective cavity gain theory which includes both the Raman and parametric contributions.

Figure 1 shows two typical optical spectra of parametric oscillations in an ultrahigh- $Q$ silica toroidal microcavity with a minor/principal diameter of $3.8 / 67 \mu \mathrm{m}$ (aspect ratio $\left.D_{p} / d=17.63\right)$. The insets in Fig. 1 show scanning electron microscope (SEM) images of the actual toroidal microcavity used in the experiment and the squared electric field obtained by a finite element modeling (FEM). The two spectra are observed under different loading conditions. A tapered optical fiber of $1-2 \mu \mathrm{m}$ diameter is mounted on a piezocontrolled translation stage having a resolution of $20 \mathrm{~nm}$ and used to couple pump and emission powers to/from the toroidal microcavity for the characterization of quality factors and the output spectrum. ${ }^{6}$ This coupling mechanism also enables precise control of cavity loading through control of the air gap between the taper and resonator. To resonantly excite the pump mode, a narrow linewidth $(<300 \mathrm{kHz})$, tunable, external-cavity laser was scanned across the pump whispering gallery mode at a repetition frequency of $100 \mathrm{~Hz}$. In the upper graph, the signal and idler (including the subsidiary signal and idler modes) are shifted by one free spectral range Downloaded 14 Dec 2005 to 131.215.225.171. Redistribution subject
(FSR) from the pump mode. However, as can be seen in the lower spectrum, the lowest threshold signal and idler modes need not be the most adjacent modes to the pump. In certain cases, signal and idler waves offset by nine times of the FSR with respect to the pump mode have been observed within the same toroidal microcavity of Fig. 1. All the signal and idler oscillation wavelengths (including the pump mode) correspond to the fundamental TE modes (which have smaller nonlinear effective mode area than TM modes: $A_{\mathrm{eff}}^{\mathrm{TE}}<A_{\mathrm{eff}}^{\mathrm{TM}}$ ) of the toroidal microcavity and the corresponding FSR for these modes is $\sim 7.8 \mathrm{~nm}$ near $1550 \mathrm{~nm}$. The minimum intrinsic $Q$ factor throughout the experiments was $0.7 \times 10^{8}$.

Figure 2 illustrates two distinct regimes of operation with respect to frequency detuning, defined by $\Delta f=2 f_{p}-f_{s}$ $-f_{i}$, where $f_{p}, f_{s}$, and $f_{i}$ are pump, signal, and idler mode frequencies, respectively. These regimes are observable within the same microcavity by selection of pump wavelength. Frequency detuning, which results from both material dispersion (in this case, thermally grown silica) as well as cavity mode dispersion (intrinsic FSR changes as a function of wavelength), will vary with wavelength and hence with the pump wavelength. In each graph, the input pump power was always fixed below $\sim 1 \mathrm{~mW}$ and only the cavity loading has been changed by varying the tapered fiber position. In Fig. 2(a), the transition from Raman oscillation to parametric oscillation (as the cavity loading was varied gradually from undercoupled to overcoupled regimes) is clearly demonto AIP license or copyright, see http://apl.aip.org/apl/copyright.jsp 


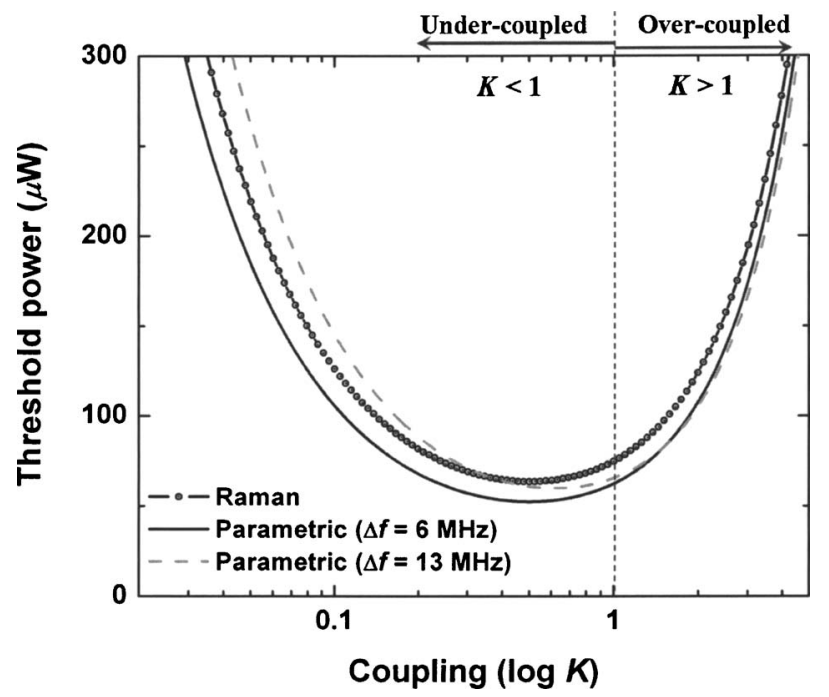

FIG. 3. (Color online) Theoretical threshold powers as a function of cavity loading ( $K<1$ : undercoupled regime; $K=1$ : critical coupling; $K>1$ : overcoupled regime). The Raman oscillation threshold along with parametric thresholds for two different frequency detuning values $(\Delta f=6$ and $13 \mathrm{MHz})$ are plotted for a toroidal microcavity with a principal/minor diameter of $67 / 3.8 \mu \mathrm{m}$. An intrinsic $Q$ factor of $10^{8}$ is assumed for the analysis.

strated for a toroidal microcavity with an estimated frequency detuning of $13 \mathrm{MHz}$ (gap width $w_{g}$ is plotted relative to the critically coupled condition: negative and positive values correspond to over- and undercoupled regimes, respectively). This kind of transition from Raman to parametric oscillation is a clear signature of intermediate to large detuning frequency values as previously predicted by the minimum threshold selection rule. ${ }^{3}$ In contrast to the large detuning case, we observe only parametric oscillation for another detuning condition $(\Delta f=6 \mathrm{MHz})$ shown in Fig. 2(b). This data is consistent with a smaller detuning frequency. ${ }^{3} \mathrm{Al}-$ though not shown explicitly in Fig. 2, a Raman-only regime can be observed within the limit of an available pump power from the same toroidal microcavity using a different pump wavelength (and, presumably, large detuning frequency).

For a semiquantitative description of the transition between these two oscillation regimes, the theory of $\chi^{(3)}$-parametric amplification including the competing Raman effect is adapted to the toroidal microresonator geometry. ${ }^{7}$ The undepleted pump-power approximation is used and relevant parameters for silica are taken from Ref. 7. For simplicity, the imaginary part of the nonlinear coefficient was assumed to be an odd function of frequency. Figure 3 shows the theoretical threshold powers as a function of cavity loading for an ultrahigh- $Q$ silica toroidal microcavity with a minor/principal diameter of 3.8/67 $\mu \mathrm{m}$. Two different frequency detuning values, $\Delta f=6$ and $13 \mathrm{MHz}$, are assumed for the calculation of parametric oscillation threshold powers. As can be seen in the graph, for small frequency detuning $[\Delta f=6 \mathrm{MHz}$, corresponding to Fig. 2(b)], the parametric oscillation threshold power is always lower than the Raman threshold. However, for an intermediate frequency detuning [ $\Delta f=13 \mathrm{MHz}$ corresponding to Fig. 2(a)], the Raman threshold power is lower than the parametric threshold in the undercoupled regime $(K<0.37)$ and becomes higher in the rest of the coupling condition resulting in a transition to the parametric oscillation. The empirical condition $D_{p} / d>15$ has

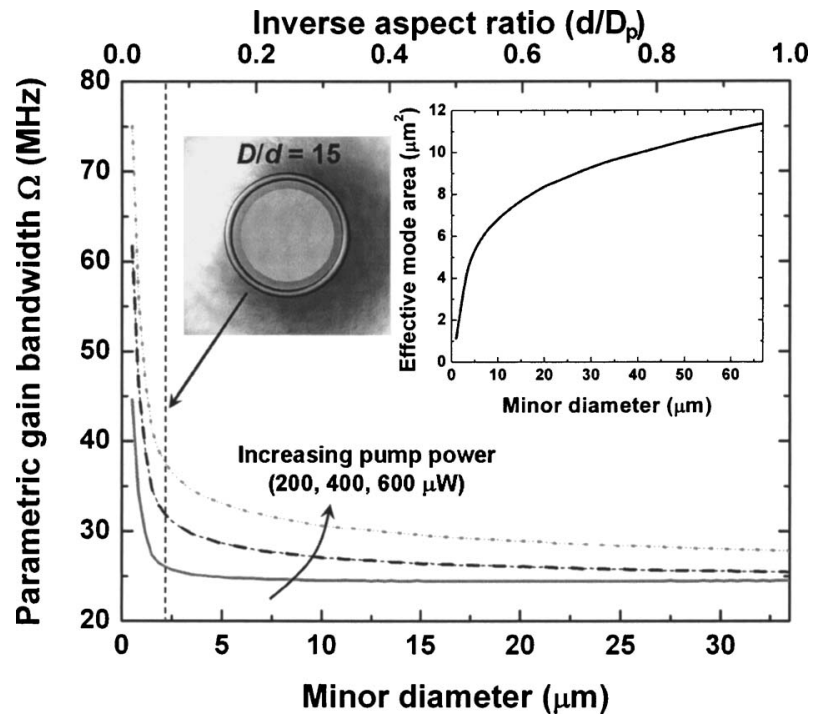

FIG. 4. (Color online) Theoretical plot of a parametric bandwidth (defined as a 3-dB effective gainbandwidth) as a function of minor diameter for three different input pump powers. Note a sharp increase in the bandwidth for a larger aspect ratio over $\sim 15$. The inset is a nonlinear effective mode area for a toroidal microcavity with a principal diameter of $67 \mu \mathrm{m}$.

also been tested with the effective cavity gain theory. In Fig. 4, the parametric gain bandwidth is plotted as a function of minor diameter for three different input pump powers. As can be seen in this plot, parametric gain bandwidth increases sharply for a larger aspect ratio exceeding $\sim 15$ thereby confirming the feasibility of the empirical number given in Ref. 3 .

In conclusion, a cavity-loading-induced transition between Raman and parametric oscillations in ultrahigh- $Q$ silica toroidal microcavities is demonstrated experimentally and theoretically analyzed using a model that incorporates both Raman and parametric effects. Frequency detuning and cavity loading are shown to play significant roles in the operation of the microcavity nonlinear oscillator in addition to the previously proven method of aspect ratio control. For a toroidal microcavity with large-enough aspect ratio $\left(D_{p} / d\right.$ $>15)$, it is possible to observe various oscillation regimes ranging from parametric to Raman in the same toroidal microcavity by direct change of cavity loading conditions or by indirect change of frequency detuning through adjustment of the pump wavelength.

This work was supported by the DARPA, NSF, and the Caltech Lee Center for Advanced Networking.

${ }^{1}$ D. K. Armani, T. J. Kippenberg, S. M. Spillane, and K. J. Vahala, Nature (London) 421, 925 (2003).

${ }^{2}$ T. J. Kippenberg, S. M. Spillane, B. Min, and K. J. Vahala, IEEE J. Sel. Top. Quantum Electron. 84, 1219 (2004).

${ }^{3}$ T. J. Kippenberg, S. M. Spillane, and K. J. Vahala, Phys. Rev. Lett. 93, 083904 (2004).

${ }^{4}$ J. J. Baumberg, P. G. Savvidis, R. M. Stevenson, A. I. Tartakovskii, M. S. Skolnick, D. M. Whittaker, and J. S. Roberts, Phys. Rev. B 62, R16247 (2000).

${ }^{5}$ R. H. Stolen and J. E. Bjorkholm, IEEE J. Quantum Electron. 18, 1062 (1982).

${ }^{6}$ M. Cai, O. Painter, and K. Vahala, Phys. Rev. Lett. 85, 74 (2004).

${ }^{7}$ P. L. Voss and P. Kumar, J. Opt. B: Quantum Semiclassical Opt. 6, S762 (2004). 